\title{
REDE SOCIAL DE APOIO À MULHER NA AMAMENTAÇÃO
}

\author{
Giselle Carlos Santos Brandão Monte ${ }^{1}$, Luciana Pedrosa Leal², Cleide Maria Pontes ${ }^{3}$
}

\begin{abstract}
RESUMO: Esta revisão integrativa objetivou identificar evidências científicas sobre ações desenvolvidas pelos atores da rede social de apoio à mulher na amamentação. A amostra foi constituída por 28 estudos publicados em português, inglês e espanhol, entre 2002 e 2011, disponíveis na Cochrane e bases de dados MEDLINE, LILACS, IBECS. Estes estudos evidenciaram que a influência negativa das mães, avós e sogras, fundamentada no mito do leite fraco, a presença do companheiro na divisão das atividades domésticas, o acolhimento e as orientações dos profissionais de saúde podem determinar o início e a continuidade, ou não, da amamentação. Outros atores que poderão fazer parte desta rede não foram citados e, deste modo, o enfermeiro precisa desenvolver pesquisas para identificar essas pessoas e realizar estratégias de educação em saúde envolvendo todos os atores da rede social de apoio à mulher para a reconstrução e/ou construção de ações que deem suporte ao sucesso da amamentação. DESCRITORES: Aleitamento materno; Apoio social; Relações familiares; Saúde da mulher.
\end{abstract}

\section{SOCIAL SUPPORT NETWORKS FOR BREASTFEEDING WOMEN}

ABSTRACT: This integrative review aimed to present a summary of the knowledge published by nurses on the conceptions of the social identity of the person with mental suffering. It included 15 articles selected from the databases, submitted to the adaptation of the questionnaire validated by Ursi-2005, for construction of a summary table. Publications from 2001 to 2011 were included which presented nurses among their authors; for the search, the DESCRIPTORS were social identification and mental health and nursing. The results indicate that $66.7 \%$ of the phenomena studied in the articles refer to issues of ethnicity and all the publications revealed elements which contribute to the configuration of the social identity as a feeling of belonging, uprootedness, adaptation to a new culture, social support, political or historical-social happenings, stigmatization and autonomy. The absence of a theoretical conception of social identity in the studies did not stop the authors from listing contributions to the improvement of research and to nursing practices directed at people with mental suffering.

DESCRIPTORS: Social identification; Mental health; Nursing; Mental suffering.

\section{RED SOCIAL DE APOYO A LA MUJER EN EL AMAMANTAMIENTO}

RESUMEN: Esta revisión integrativa tuvo el objetivo de identificar evidencias científicas acerca de acciones desarrolladas por los actores de la red social de apoyo a la mujer en el amamantamiento. La muestra se constituyó de 28 estudios publicados en portugués, inglés y español, entre 2002 y 2011, disponibles en Cochrane y bases de datos MEDLINE, LILACS y IBECS. Estes estudios evidenciaron que la influencia negativa de las madres, abuelas y suegras, fundamentada en el mito de la leche debil, la presencia del compañero en la división de las actividades domésticas, la acogida y las orientaciones de los profesionales de salud pueden determinar el inicio y la continuidad, o no, del amamantamiento. Otros actores que podrán hacer parte de esta red no fueron citados y, de este modo, el enfermero necesita desarrollar investigaciónes para identificar esas personas y realizar estrategias de educación en salud involucrando todos los actores de la red social de apoyo a la mujer para la reconstrucción y/o construcción de acciones para dar soporte al éxito del amamantamiento.

DESCRIPTORES: Amamantamiento materno; Apoyo social; Relaciones familiares; Salud de la mujer.

${ }^{1}$ Enfermeira Obstétrica. Mestre em Enfermagem. Professora do Curso de Graduação e do Propgrama de Pós-Graduação em Enfermagem da Universidade Federal de Alagoas e do Curso de Graduação em Enfermagem da Universidade Estadual de Ciências da Saúde de Alagoas ${ }^{2}$ Enfermeira Pediátrica. Doutora em Nutrição. Professora do Departamento de Enfermagem e do Programa de Pós-Graduação em Enfermagem do Centro de Ciências da Saúde da Universidade Federal de Pernambuco CCS UFPE.

${ }^{3}$ Enfermeira Obstétrica. Doutora em Nutrição. Professora do Departamento de Enfermagem e do Programa de Pós-Graduação em Enfermagem do CCS UFPE. 


\section{INTRODUÇÃO}

Todas as pessoas fazem parte de alguma rede social, de um conjunto de relações interpessoais que vinculam indivíduos a outros indivíduos. Mesmo que de forma imperceptível e informal, os seres humanos são marcados por uma interdependência entre si, que atende a interesses e afinidades que podem ser temporários ou não ${ }^{(1)}$. Essa rede social, denominada também de suporte social ou apoio social, é composta por pessoas de confiança, pessoas que se mostram preocupadas com o outro, o valorizam, gostam do mesmo e principalmente, tem disponibilidade para ajudar ${ }^{(2)}$.

No contexto da amamentação, a mulher é influenciada pela sua rede social de apoio, uma vez que ela pode receber incentivo para amamentar, ou não, seu filho ${ }^{(3)}$. Neste sentido, a cultura e as tradições podem prevalecer em relação às orientações dos profissionais de saúde ${ }^{(4)}$. A amamentação, portanto, não é uma prática instintiva ou automática, é uma ação guiada pela subjetividade e experiência das mulheres, influenciada pelo contexto social em que vivem ${ }^{(5)}$.

Por isso, este estudo tem o objetivo de identificar evidências científicas sobre as ações desenvolvidas pelos atores da rede social de apoio à mulher na amamentação.

\section{MÉTODO}

O presente estudo consiste em uma revisão integrativa da literatura, método que possibilita a síntese do conhecimento produzido acerca de um tema, além de proporcionar a aplicabilidade de diversos resultados na prática ${ }^{(6-7)}$. A sua construção foi norteada por seis etapas: elaboração da pergunta norteadora (como as pessoas da rede social de apoio à mulher estão atuando no contexto da amamentação?), busca na literatura, coleta de dados e categorização dos estudos, análise crítica, discussão dos resultados e apresentação da revisão integrativa ${ }^{(7)}$.

Na segunda etapa (busca das publicações científicas) foram adotadas como fontes de informação o Portal da Biblioteca COCHRANE e as bases eletrônicas de dados LILACS, MEDLINE e IBECS. Os critérios de inclusão para a seleção foram: artigos publicados entre 2002 e 2011, teses e dissertações, em português, inglês e espanhol, disponíveis na íntegra e que respondessem à questão norteadora. Foram excluídos editoriais, resumos de pesquisas, avaliações econômicas e artigos de jornal isento de caráter científico.

A busca das publicações ocorreu entre novembro e dezembro de 2011, utilizando a combinação dos descritores "Aleitamento Materno", "Apoio Social" e "Relações Familiares", todos incluídos nos Descritores em Ciências da Saúde (DeCS) e suas respectivas traduções padronizadas no Medical Subject Heading (MESH): Breast Feeding, Social Support e Family Relations. Inicialmente, realizou-se a busca pelos descritores individualmente. Em seguida, foram realizados os cruzamentos, utilizando o operador booleano and, entre os descritores: Aleitamento Materno e Apoio Social; Aleitamento Materno e Relações Familiares. Por último, os três descritores foram cruzados em conjunto, conforme apresentado na tabela 1 .

Após os cruzamentos, foram encontradas 434 publicações, aquelas que se repetiram foram computadas considerando a seguinte ordem hierárquica: COCHRANE, MEDLINE, LILACS e IBECS.

Para a seleção das publicações, primeiramente foram lidos todos os títulos dos estudos. Nas 314 publicações em que os títulos possuíam alguma palavra que remetesse ao objeto da revisão integrativa, os resumos foram analisados. Entre esses, 55 apresentaram relevância para o presente estudo, sendo as publicações lidas na íntegra. Destas, foram selecionadas 28 , as quais respondiam à questão norteadora e atendiam aos critérios de inclusão determinados, estando disponíveis nas seguintes bases de dados: MEDLINE (12), LILACS (14) e no Portal COCHRANE (02). Portanto, das 434 publicações iniciais, constituíram o corpus final 27 artigos e uma dissertação de mestrado (tabelas 2 e 3 ).

$\mathrm{Na}$ terceira etapa (coleta de dados) utilizou-se um instrumento adaptado do modelo proposto por $\mathrm{Ursi}^{(8)}$, contendo: título, ano, autor, objetivos, metodologia, sujeitos, principais resultados e nível de evidência dos estudos. Os resultados, provenientes do conhecimento produzido, foram agrupados por proximidade temática $^{(7)}$.

Na quarta etapa (análise das publicações) foi avaliado o rigor metodológico, considerando clareza na descrição dos métodos empregados, participantes, critérios de inclusão/exclusão, intervenção, resultados, limitações e vieses ${ }^{(8)}$.

A quinta etapa (discussão dos resultados) está apresentada de forma descritiva, possibilitando a identificação das evidências e a necessidade de outras pesquisas para subsidiar a prática do enfermeiro direcionada ao manejo do aleitamento materno. 
Tabela 1 - Publicações encontradas entre os anos de 2002 e 2011, sobre 'Rede de apoio à mulher na amamentação'. Recife, 2011

\begin{tabular}{lccccc}
\hline Descritores & MEDLINE & LILACS & IBECS & COCHRANE & Total \\
\hline $\begin{array}{l}\text { Aleitamento materno and } \\
\text { Apoio Social }\end{array}$ & 360 & 29 & 0 & 8 & 397 \\
$\begin{array}{l}\text { Aleitamento Materno and } \\
\text { Relações Familiares }\end{array}$ & 28 & 7 & 1 & 1 & 37 \\
$\begin{array}{l}\text { Aleitamento Materno and } \\
\text { Apoio Social and Relações }\end{array}$ & 0 & 0 & 0 & 0 & 0 \\
$\begin{array}{l}\text { Familiares } \\
\text { Total }\end{array}$ & 388 & 36 & 1 & 9 & 434 \\
\hline
\end{tabular}

Tabela 2. Publicações excluídas de acordo com os critérios de inclusão estabelecidos, relativas aos anos de 2002 a 2011. Recife, 2011

\begin{tabular}{lccccc}
\hline Critérios & MEDLINE & LILACS & IBECS & COCHRANE & Total \\
\hline $\begin{array}{l}\text { Resumos sem relevância } \\
\text { para o estudo }\end{array}$ & 243 & 13 & 0 & 3 & 259 \\
$\begin{array}{l}\text { Fora do período } \\
\text { estabelecido }\end{array}$ & 108 & 0 & 0 & 0 & 108 \\
$\begin{array}{l}\text { Não responde à pergunta } \\
\text { norteadora }\end{array}$ & 19 & 4 & 1 & 3 & 27 \\
$\begin{array}{l}\text { Repetidos } \\
\text { Total excluído }\end{array}$ & 6 & 5 & 0 & 1 & 12 \\
\hline
\end{tabular}

Tabela 3. Publicações selecionadas sobre 'Rede de apoio à mulher na amamentação'. Recife, 2011

\begin{tabular}{lcccc}
\hline Fonte & Publicações & Resumos & Leitura na íntegra & Publicações Selecionadas \\
\hline MEDLINE & 388 & 274 & 31 & 12 \\
LILACS & 36 & 31 & 18 & 14 \\
IBECS & 1 & 1 & 1 & 0 \\
COCHRANE & 9 & 8 & 5 & 2 \\
Total & 434 & 314 & 55 & 28 \\
\hline
\end{tabular}

\section{RESULTADOS}

Em relação ao idioma de publicação, o português predominou (19), seguido do inglês (8) e espanhol (1). Quanto ao ano de publicação, 2008 apresentou o maior número de estudos (8) e a maioria destes (14) foi identificada na LILACS, seguida da MEDLINE (12), conforme tabela 3. Entre os autores destes estudos destacam-se médicos (37) e enfermeiros (30).

Nos estudos analisados observou-se o mesmo número (13) desenvolvido no método quantitativo e qualitativo, além de uma revisão integrativa da literatura e uma revisão bibliográfica. $\mathrm{Na}$ abordagem quantitativa foram selecionados um artigo de revisão sistemática, dois estudos de avaliação de serviços, quatro estudos prospectivos e seis ensaios clínicos randomizados. Em quatro desses últimos, o profissional enfermeiro estava entre os autores.
Dentro da classificação hierárquica metodológica proposta pela Prática Baseada em Evidências ${ }^{(9)}$, foi classificada como estudo com nível 1 de evidência a revisão sistemática de ensaios clínicos controlados e randomizados. Apenas seis dos artigos foram classificados no nível 2 de evidência, o qual corresponde a estudos individuais com delineamento experimental, randomizados e controlados; outros dois estudos foram classificados como nível 5 de evidência, os quais referem-se a dados de avaliação de serviços. A maioria apresentou nível 4, os quais são caracterizados por evidências de estudos descritivos (não-experimentais) ou com abordagem qualitativa.

O quadro 1 mostra uma sinopse dos principais resultados encontrados que, de um modo geral, identificaram a mãe, a avó, o companheiro e os profissionais de saúde como principais atores da rede social de apoio à mulher. 
Quadro 1 - Sinopse dos principais resultados encontrados nas publicações selecionadas entre 2002 e 2011 sobre 'Rede social de apoio à mulher na amamentação'. Recife, 2011

\begin{tabular}{|l|c|c|}
\hline Agrupamento dos resultados & Publicações & Referências \\
\hline $\begin{array}{l}\text { Cultura popular/familiar como determinante das práticas do aleitamento } \\
\text { materno em detrimento ao apoio dos profissionais de saúde }\end{array}$ & 4 & $10,13,29-30$ \\
\hline $\begin{array}{l}\text { Rede social de apoio à mulher sendo favorecedora do processo do } \\
\text { aleitamento materno }\end{array}$ & 11 & $11-18,23,25,31,35$ \\
\hline $\begin{array}{l}\text { Práticas educativas exercidas pelos profissionais de saúde no pré e } \\
\text { pós-natal como elemento favorecedor, ou não, para o início e manu- } \\
\text { tenção do aleitamento materno }\end{array}$ & 10 & $19-22,24,36-39,41$ \\
\hline $\begin{array}{l}\text { Fatores que levaram ao desmame precoce e/ou atores da rede social } \\
\text { que dificultaram o aleitamento materno }\end{array}$ & 3 & $26-28$ \\
\hline
\end{tabular}

\section{DISCUSSÃO}

O aleitamento materno é uma atividade complexa para a mulher, pois não envolve apenas o querer amamentar; é um entrelaçamento do físico, do psíquico e do contexto social da mulher ${ }^{(10)}$. Portanto, o apoio de sua rede social é essencial para o início e continuidade do aleitamento materno exclusivo até os seis meses e com complementação até os dois anos ou mais de vida da criança ${ }^{(11-24)}$, conforme preconiza o Ministério da Saúde do Brasil. Desse modo, conhecer o cenário vivenciado pela mulher, seu ciclo de convivência e os fatores que interferem na sua decisão de amamentar deveria ser considerado em todos os momentos da assistência prestada a ela e sua família, nos serviços de saúde e domicílio, durante o ciclo gravídico-puerperal ${ }^{(19,25)}$.

A rede social de apoio faz parte desse cenário, considerada um conjunto de relações que determinam as características da pessoa, tais como hábitos, costumes, crenças e valores. Esta rede, somada à maior vulnerabilidade da mulher às influências múltiplas, devido à maternidade e ao processo de lactação, exerce interferência na decisão da mulher em amamentar, ou não ${ }^{(26)}$.

No entanto, a análise dos artigos evidenciou que, dentro da rede social de apoio à mulher, as pessoas próximas a ela principalmente as avós (maternas e paternas) podem exercer influência negativa na prática da amamentação, tanto na sua duração quanto no tipo de aleitamento materno. Isso ocorre devido à sua vivência com a amamentação, envolvida pelas crenças, mitos e tabus existentes, os quais são muito mais impactantes que as orientações recebidas dos profissionais ${ }^{(10,13,15,26-30)}$, pois na maioria das vezes as suas filhas desmamam precocemente seus netos.

Resulta que uma figura feminina, que já tenha sido mãe, é percebida por outra mulher de maneira significa- tiva, por ter vivenciado as dificuldades, os medos e os anseios relativos à maternidade $\mathrm{e}$ ao aleitamento materno ${ }^{(27)}$. A filha toma a mãe como exemplo a ser seguido, copiado e transformado. Até o fato de 'ter leite' é determinado pelas pessoas que são importantes para a mulher-nutriz, as quais podem ajudar, ou não, na produção láctea, devido ao apoio fornecido durante os momentos de dificuldade ${ }^{(27)}$.

Outra pessoa da rede social que demonstrou ser essencial no processo do aleitar foi o pai/companheiro. A sua participação, muitas vezes considerada nula, pode ser decisória para o início e continuidade da amamentação, através do incentivo, do estar junto da mulher e, até mesmo, dividindo as atividades domésticas e os cuidados com os filhos mais velhos. Dessa maneira, a mulher sente-se valorizada por estar amamentando e pode ter mais tempo para se dedicar ao recém-nascido ${ }^{(11,14-15,17,23,25,31-32)}$.

Além disso, a ausência de apoio oferecido pelos profissionais de saúde também foi relatada como determinante para o desmame precoce. É importante ressaltar que o profissional de saúde, incluindo o enfermeiro, deve apoiar e incentivar a lactante para a prática do aleitamento materno. Neste sentido, preparando-a emocionalmente, informando-a sobre a fisiologia da lactação, seus benefícios, o cuidado com as mamas, o correto posicionamento dela e do bebê durante a amamentação, devendo este preparo ser iniciado nas consultas de pré-natal e perdurar até as visitas domiciliares do pós-parto ${ }^{(10-11,13-15,18-25)}$.

No entanto, no cotidiano das práticas assistenciais, os profissionais de saúde tendem a realizar orientações bancárias, as quais não permitem a construção de consciência crítica e de educação dialógica problematizadora e participante ${ }^{(33)}$; biologizantes, voltadas apenas para o corpo biológico ${ }^{(34)}$; técnicas, fundamentadas nas necessidades da criança, omitindo outras vantagens e algumas dificuldades que podem ocorrer durante a amamentação. Esta omissão pode levar as mulheres a 
procurar esclarecimentos com pessoas que não as orientam corretamente. Esta possibilidade pode contribuir na interrupção precoce da amamentação.

O engajamento e comprometimento desses profissionais em ações de educação em saúde, com metodologias ativas, humanizadas e integrais, pode aumentar os índices de aleitamento materno. No entanto, chama atenção que alguns resultados dos estudos analisados demonstram que grupos de apoio à amamentação, principalmente aqueles realizados durante a gestação, não impulsionaram o aumento na duração do período do aleitamento materno. Isso pode estar atrelado às estratégias realizadas, as quais, muitas vezes, são direcionadas pela transmissão vertical das informações que desconsidera o saber prévio e não permite às usuárias a oportunidade de participar efetivamente ${ }^{(33-39)}$. É necessário que os profissionais de saúde reflitam sobre sua atitude, compreendendo o processo de aleitar inserido no contexto das mulheres e de sua família, considerando as dificuldades e limitações de cada uma para, em seguida, elaborar um plano estratégico que contemple a realidade vivenciada.

Desse modo, o diagnóstico precoce da realidade das mulheres identifica suas necessidades de saúde, sua cultura e o seu meio, sendo fundamental para a elaboração das estratégias de educação em saúde. Nesse sentido, criam-se possibilidades de maior participação e colaboração por parte das usuárias, podendo propiciar ações mais eficazes e humanísticas ${ }^{(40)}$. Do contrário, sem esse envolvimento, não ocorrerá uma ação transformadora e sim uma ação imposta pelo profissional de saúde, para a qual, na maioria das vezes, não há continuidade.

Estratégia interessante demonstrada por um dos estudos foi a utilização da linha telefônica como meio de comunicação entre as puérperas e os profissionais de saúde, para esclarecimentos de dúvidas quanto aos cuidados com o recém-nascido, a mulher e a amamentação. Os resultados positivos desta ação foram comprovados pela satisfação das mulheres alicerçada no apoio e acolhimento recebidos ${ }^{(21)}$. Além de ser uma maneira simples de aproximar a assistência à realidade e a necessidade de cada mulher, pode colaborar para aumentar a prevalência do aleitamento materno.

Outros estudos enfatizaram que o retorno da mulher ao trabalho/estudo pode ocasionar dificuldades para a prática da amamentação. As mulheres que trabalham fora do lar, ou que têm essa intenção, não conseguem manter o aleitamento materno, pois as empresas/escolas/faculdades não possuem ambiente adequado para a ordenha e o armazenamento do leite, ou a preocupação com os horários que o bebê precisa mamar. Dessa forma, as lactantes oferecem outros tipos de alimentos para saciar a fome do seu filho, iniciando o desmame precocemente ${ }^{(11,26,41)}$.

Assim, entende-se que o apoio da rede social é essencial para a continuidade do amamentar, servindo como suporte nesse período de vida. Portanto, toda teia de significados que envolvem esta rede deve ser valorizada, inclusive os sentimentos das pessoas que apresentam vínculo próximo com a mulher nutriz, geralmente a mãe, para que o ato de amamentar transcorra de forma efetiva ${ }^{(10-11,15-17,27-28)}$.

\section{CONCLUSÃO}

As evidências científicas mostraram que as pessoas da rede social de apoio à mulher, presentes no seu cotidiano, principalmente a sua mãe, a avó, a sogra e o companheiro, interferem na prática da amamentação. Essa influência é maior quanto maior for o tempo de convivência.

De acordo com os estudos, esses atores, inclusive os profissionais de saúde, podem contribuir, ou não, para o início e manutenção da amamentação, dependendo das ações desenvolvidas por eles. Entre estas ações merecem destaque a influência das mães, avós e sogras devido às crenças; a presença do companheiro na divisão das atividades domésticas e no cuidados com os filhos mais velhos; o apoio, o acolhimento, o esclarecimento de dúvidas e as orientações dos profissionais de saúde, muitas vezes realizadas em grupos de gestantes. Por outro lado, ressalta-se que a ausência de apoio de algum desses atores poderá ocasionar o desmame precoce. Neste contexto, este estudo contribui para a tomada de decisão do enfermeiro na realização de estratégias de educação em saúde direcionadas ao apoio à nutriz e outras pessoas que fazem parte da rede social da mulher para aumentar a duração do período do aleitamento materno.

Esta revisão constatou ainda que a família extensiva (tios, primos, agregados, amigos, vizinhos) e outras pessoas da comunidade (líderes comunitários, benzedeiras, pastores) não foram citadas nos estudos selecionados. Esta lacuna limita a identificação de novos indivíduos que compõem a rede social de apoio à mulher. Assim, novas pesquisas devem ser desenvolvidas para identificar outros atores desta rede que poderão ajudar a mulher durante a lactação. Neste sentido, estudos experimentais e quase-experimentais, centrados em ações educativas desenvolvidas no campo da saúde e da enfermagem, pre- 
cisam ser realizados para buscar as melhores evidências para envolver de forma efetiva a rede social de apoio para o sucesso do aleitamento materno.

\section{REFERÊNCIAS}

1. Braga, NA. Redes sociais de suporte e humanização dos cuidados em saúde. In: Deslandes SF, Moreira MEL, editores. Humanização dos cuidados em Saúde: Conceitos, dilemas e práticas. Rio de Janeiro: Fiocruz; 2006. p. 163-83.

2. Gonçalves TR, Pawlowski J, Bandeira DR, Piccinini CA. Avaliação de apoio social em estudos brasileiros: aspectos conceituais e instrumentos. Ciênc. saúde colet. [Internet] 2011;16(3) [acesso em 03 nov 2011]. Disponível: http://www.scielo.br/pdf/ csc/v16n3/12.pdf

3. Turnbull-Plaza B, Escalante-Izeta E, KlunderKlunder M. The role of social networks in exclusive breastfeeding. Rev Med Inst Mex Seguro Soc. [Internet] 2006; 44(2) [acesso em 12 nov 2011]. Disponível: http:// www.ncbi.nlm.nih.gov/pubmed/16674855

4. Parizotto J, Zorzi NT. Aleitamento materno: fatores que levam ao desmame precoce no município de Passo Fundo, RS. Mundo Saúde. [Internet] 2008;32(4) [acesso em 03 nov 2011]. Disponível: http://www.saocamilo-sp. br/pdf/mundo_saude/65/08_Aleitamento_baixa.pdf

5. Marques ES, Cotta RMM, Botelho MIV, Franceschini SCC, Araújo RMA, Lopes LL. Rede social: desvendando a teia de relações interpessoais da nutriz. Physis [Internet] 2010;20(1) [acesso em set 2011]. Disponível: http://www.scielo.br/pdf/physis/v20n1/ a14v20n1.pdf

6. Souza MT, Silva MD, Carvalho R. Revisão integrativa: o que é e como fazer. einstein. 2010;8(1):102-6.

7. Mendes KDS, Silveira RCCP, Galvão CM. Revisão integrativa: método de pesquisa para a incorporação de evidências na saúde e na enfermagem. Texto ContextoEnferm. 2008;17(4):758-64.

8. Ursi ES. Prevenção de lesões de pele no perioperatório: revisão integrativa da literatura. [dissertação]. Ribeirão Preto (SP): Universidade de São Paulo; 2005.

9. Galvão CM. Editorial. Níveis de evidência. Acta Paul Enferm [Internet] 2006;19(2) [acesso em 20 out 2012]. Disponível: http://www.scielo.br/pdf/ape/v19n2/ a01v19n2.pdf
10. Teixeira MA, Nitschke RG, Gasperi P, Siedler MJ. Significados de avós sobre a prática do aleitamento materno no cotidiano familiar: a cultura do quererpoder amamentar. Texto Contexto-Enferm. [Internet] 2006;15(1) [acesso em 03 nov 2011]. Disponível: http:// www.scielo.br/pdf/tce/v15n1/a12v15n1.pdf

11. Müller FS, Silva IA. Social representations about support for breastfeeding in a group of breastfeeding women. Rev. Latino-Am. Enfermagem. 2009;17(5):651-7.

12. Souza MHN, Souza IEO, Tocantins FR. Abordagem da fenomenologia sociológica na investigação da mulher que amamenta. Rev. enferm. UERJ. [Internet] 2009;17(1) [acesso em 15 dez 2011]. Disponível: http:// www.facenf.uerj.br/v17n1/v17n1a10.pdf

13. Braga DF, Machado MMT, Bosi MLM. Amamentação exclusiva de recém-nascidos prematuros: percepções e experiências de lactantes usuárias de um serviço público especializado. Rev. Nutr. [Internet] 2008;21(3) [acesso em 15 dez 2011]. Disponível: http://www.scielo. br/pdf/rn/v21n3/a04v21n3.pdf

14. Buchala LM, Moraes MS. Amamentação vivenciada com sucesso por um grupo de mulheres. Arq. Ciênc. saude. [Internet] 2005;12(4) [acesso em 15 dez 2011]. Disponível: http://www.cienciasdasaude.famerp.br/ racs_ol/vol-12-4/03_ID131.pdf

15. Marques ES, Cotta RMM, Magalhães KA, Sant'Ana FR, Gomes AP, Batista RS. A Influência da rede social da nutriz no aleitamento materno:o papel estratégico dos familiares e dos profissionais de saúde. Ciênc. saúde colet. [Internet] 2010;15(Supl. 1) [acesso em 12 nov 2011]. Disponível: http://www.scielo.br/pdf/csc/v15s1/049.pdf

16. Morgado CMC. Rede e apoio social e práticas alimentares de crianças no quarto mês de vida [dissertação]. Rio de Janeiro (RJ): Universidade do Estado do Rio de Janeiro; 2009.

17. Souza MHN, Souza IEO, Tocantins FR. A Utilização do referencial metodológico de rede social na assistência de enfermagem a mulheres que amamentam. Rev. Latino-Am. Enfermagem. [Internet] 2009;17(3) [acesso em 15 dez 2011]. Disponível: http://www.scielo.br/pdf/rlae/v17n3/pt_12.pdf

18. Britton C, McCormick FM, Renfrew MJ, Wade A, King SE. Apoyo para la lactancia materna. Reproducción de una revisión Cochrane, traducida y publicada en La Biblioteca Cochrane Plus. [Internet] 2008;(2) [acesso em $18 \mathrm{dez} 2011$ ]. Disponível: http:// www.update software.com/BCP/BCPGetDocument. asp?DocumentID $=\mathrm{CD} 001141$ 
19. Su L-L, Chong Y-S, Chan Y-H, Chan Y-S, Fok D, Tun $\mathrm{K}-\mathrm{T}$, et al. Antenatal education and postnatal support strategies for improving rates of exclusive breast feeding: randomized controlled trial. BMJ. [Internet] 2007 [acesso em 18 dez 2011]. Disponível: http://www. ncbi.nlm.nih.gov/pmc/articles/PMC1989016/pdf/bmj335-7620-res-00596-el.pdf

20. Albernaz E, Araújo CL, Tomasi E, Mintem G, Giugliani E, Matijasevich A, et al. Influência do apoio à amamentação nas tendências das taxas de aleitamento materno da cidade de Pelotas (RS), 1982-2004. J. Pediatr. [Internet] 2008;84(6) [acesso em $15 \mathrm{dez} 2011$ ]. Disponível: http://www.scielo.br/pdf/jped/v84n6/ en_v84n6a15.pdf

21. Osman H, Chaaya M, Zein LE, Naassan G, Wick L. What do first-time mothers worry about? A study of usage patterns and content of calls made to a postpartum support telephone hotline. BMC Public Health. [Internet] 2010;10(611) [acesso em 18 dez 2011]. Disponível: http://www.biomedcentral.com/content/ pdf/1471-2458-10-611.pdf

22. Dennis C-L, Hodnett E, Gallop R, Chalmers B. The effect of peer support on breast-feeding duration among primiparous women: a randomized controlled trial. Can Med Assoc J [Internet] 2002;166(1) [acesso em $18 \mathrm{dez}$ 2011]. Disponível: http://www.canadianmedicaljournal. ca/content/166/1/21.full.pdf

23. Machado MMT, Bosi MLM. Compreendendo a prática do aleitamento exclusivo: um estudo junto a lactantes usuárias da Rede de Serviços em Fortaleza, Ceará, Brasil. Rev. Bras. Saude Mater. Infant. [Internet] 2008;8(2) [acesso em 09 nov 2011]. Disponível: http:// www.scielo.br/pdf/rbsmi/v8n2/06.pdf

24. Oliveira MIC, Camacho LAB, Souza IEO. Promoção, proteção e apoio à amamentação na atenção primária à saúde no Estado do Rio de Janeiro, Brasil: uma política de saúde pública baseada em evidência. Cad. Saúde Pública. [Internet] 2005;21(6) [acesso em 15 dez 2011]. Disponível: http://www.scielo.br/pdf/csp/v21n6/30.pdf

25. Pinto TV. Promoção, protecção e apoio ao aleitamento materno na comunidade: revisão das estratégias no período pré-natal e após a alta. Arq. Med. [Internet] 2008;22(2/3) [acesso em 12 nov 2011]. Disponível: http://www.scielo. oces.mctes.pt/pdf/am/v22n2-3/22n2-3a05.pdf

26. Ramos CV, Almeida JAG. Alegações maternas para o desmame: estudo qualitativo. J. pediatr. [Internet] 2003;79(5) [acesso em 03 nov 2011]. Disponível: http:// www.scielo.br/pdf/jped/v79n5/v79n5a04.pdf
27. Susin LRO, Giugliani ERJ, Kummer SC. Influência das avós na prática do aleitamento Materno. Rev. Saúde Pública. [Internet] 2005;39(2) [acesso em 15 dez 2011]. Disponível: http://www.scielosp.org/pdf/rsp/ v39n2/24034.pdf

28. Ichisato SMT, Shimo AKK. Vivência da amamentação: lactogogos e rede de suporte. Cienc. cuid. saude. [Internet] 2006;5(3) [acesso em 03 nov 2011]. Disponível: http://www.periodicos.uem.br/ojs/index. php/CiencCuidSaude/article/view/5052/3258

29. Teixeira MA, Nitschke RG. Modelo de cuidar em enfermagem junto às mulheres-avós e sua família no cotidiano do processo de amamentação. Texto Contexto-Enferm. [Internet] 2008;17(1) [acesso em 13 nov 2011]. Disponível: http://www.scielo.br/pdf/tce/ v17n1/21.pdf

30. Machado ARM, Nakano AMS, Almeida AM, Mamede MV. O lugar da mãe na prática da amamentação de sua filha nutriz: o estar junto. Rev Bras Enferm. [Internet] 2004;57(2) [acesso em 12 nov 2011]. Disponível: http:// www.scielo.br/pdf/reben/v57n2/a10v57n2.pdf

31. Piazzalunga CRC, Lamounier JA. O contexto atual do pai na amamentação: uma abordagem qualitativa. Rev Med Minas Gerais. [Internet] 2011;21(2) [acesso em 09 nov 2011]. Disponível: http://rmmg.medicina.ufmg.br/ index.php/rmmg/article/viewFile/361/346

32. Cruz DSM, Rocha INSBS, Marques DKA, Souza IVB. Percepção da figura paterna frente ao aleitamento materno. Cogitare enferm. [Internet] 2011;16(4) [acesso em 10 abr 2012]. Disponível: http://ojs.c3sl.ufpr.br/ojs2/ index.php/cogitare/article/viewFile/25441/17063

33. Freire P. A pedagogia do oprimido. $36^{\mathrm{a}}$ ed. Rio de Janeiro: Edições Paz e Terra; 2003.

34. Chagas NR, Ramos IC, Silva LF, Monteiro ARM, Fialho AVM. Cuidado crítico e criativo: contribuições da educação conscientizadora de Paulo Freire para a enfermagem. Ciencia Y Enfermeria [Internet] 2009;XV(2) [acesso em 09 out 2012]. Disponível: http:// www.scielo.cl/pdf/cienf/v15n2/art05.pdf.

35. Hoddinott P, Britten J, Prescott G J, Tappin D, Ludbrook A, Godden D J. Effectiveness of policy to provide breastfeeding groups (BIG) for pregnant and breastfeeding mothers in primary care: cluster randomised controlled trial. BMJ. [Internet] 2009;338 [acesso em 15 dez 2011]. Disponível:http://www.bmj. com/highwire/filestream/339898/field_highwire_ article_pdf/0.pdf 
36. Oliveira MIC, Camacho LAB. Impacto das unidades básicas de saúde na duração do aleitamento materno exclusivo. Rev. bras. epidemiol. [Internet] 2002;5(1) [acesso em 09 nov 2011]. Disponível: http://www. scielosp.org/pdf/rbepid/v5n1/06.pdf

37. Graffy J, Taylor J, Williams A, Eldridge S. Randomised controlled trial of support from volunteer counselors for mothers considering breast feeding. BMJ. [Internet] 2004;328 [acesso em 18 dez 2011]. Disponível: http:// www.ncbi.nlm.nih.gov/pmc/articles/PMC313903/pdf/ bmj32800026.pdf

38. MacArthur C, Jolly K, Ingram L, Freemantle N, Dennis C-L, Hamburger R, et al. Antenatal peer support workers and initiation of breast feeding: cluster randomised controlled trial. BMJ. [Internet] 2008;338 [acesso em 12 nov 2011]. Disponível: http://www.bmj. com/highwire/filestream/348396/field_highwire article_pdf/0.pdf

39. Muirhead PE, Butcher G, Rankin J, Munley A. The effect of a programme of organised and supervised peer support on the initiation and duration of breastfeeding: a randomised trial. Br J Gen Pract. [Internet] 2006;56 [acesso em 03 nov 2011]. Disponível: http://www.ncbi.nlm. nih.gov/pmc/articles/PMC1828262/pdf/bjpg56-191.pdf

40. Boehs AE, Monticelli M, Wosny AM, Heidemann IBS, Grisotti M. A interface necessária entre enfermagem, educação em saúde e o conceito de cultura. Texto Contexto-Enferm. [Internet] 2007;16(2) [acesso em 09 nov 2011]. Disponível: http://www.scielo.br/pdf/tce/ v16n2/a14v16n2.pdf

41. Silva IA. A vivência de amamentar para trabalhadoras e estudantes de uma universidade pública. Rev Bras Enferm. [Internet] 2005;58(6) [acesso em 15 dez 2011]. Disponível: http://www.scielo.br/pdf/reben/v58n6/ a03v58n6.pdf 